\title{
Research on Moving Target Detection Algorithm in Mine Safety Monitoring
}

\author{
Yulian Chen \\ Lanzhou Resource and Environment Voc-Tech College, Electrical and Mechanical Department, Lanzhou, \\ 730021, China
}

Keywords: Mine Safety, Monitoring Center, Moving Target, Detection Algorithm, Research

\begin{abstract}
Mine safety problem is directly related to the personal health of the staff, it needs to strengthen the safety control, which is very important at present, in order to ensure that the staff can work safely in the underground environment, the safety monitoring system appears, this system can check the working environment, the staff's performance in the dangerous area is tracked and tracked, according to the activities of the workers, analyze whether it is safe to work underground, under the system analysis, can grasp the safety of the working environment in time. The security monitoring system combines the early warning function with the linkage system organically, so that the work can achieve good results and can effectively prevent the occurrence of danger during the work.
\end{abstract}

\section{Introduction}

In order to improve the performance of the system, the system is further optimized, combining with the color gradient and so on, to construct the gaussian mathematical model. The safety monitoring system is a system which uses the light to work, so it can use the line to adjust the learning rate to match the color gradient in the system, which can further improve the safety monitoring performance of the mine.

\section{Research Background}

Coal mining resources are important resources, but coal mining is very difficult at present, and the frequent occurrence of safety accidents has seriously affected the effect of work execution. In order to solve this problem effectively, attention should be paid to the safety inspection work, which will affect the development of national economy, and the safety video monitoring system will effectively solve the problems of complex environment, changeable safety and low coefficient of underground.

\section{System Composition}

In order to improve the safety factor of the track transportation of the inclined lane winch, it is necessary to do a good job of safety monitoring, which can effectively avoid the safety accidents in the work, use the safety video monitoring system to monitor the working environment and the working conditions of the workers in the process of operation and transportation. The safety video monitoring system can freely switch the digital signals and video signals through the video service technology to understand the working conditions of the underground workers through the signal transmission. In the display, it can effectively avoid the occurrence of the fault of the lane track transportation and the safety hidden danger.

In the past, it is difficult for the winch driver to respond quickly in a short time under the complicated environment, so it will increase the danger invisibly, the staff should know this situation as soon as possible, adopt more scientific way, use the image to prompt the road condition, it is a more reasonable scheme, because the downhole operation can solve the problem very well because the equipment or the staff form the big noise. The explosion-type switch enables the digital signal and the video signal to switch freely, thus can play the image data through the security video server, in the information network development process, the information transmission becomes very 
simple, many information can transmit in a short time, the digital signal becomes the video signal, then can broadcast the underground work situation on the video interface through the authorized computer permission, then can expand the monitoring scope, the manager can understand the underground operation situation on the ground, the real-time monitoring can reduce the work efficiency to a great extent, and ensure the life safety of the staff.

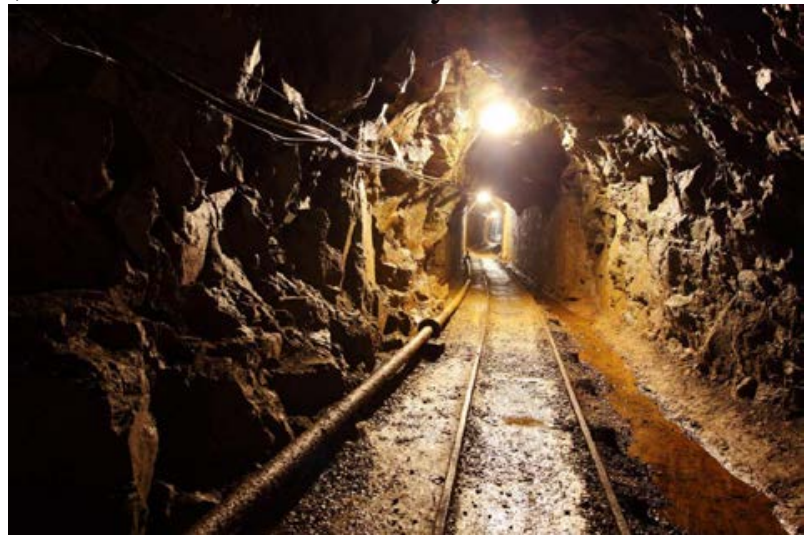

Figure 1 Working chart of mine monitoring system

\section{Analysis of Current Mine Monitoring System Algorithm}

The existing algorithms of the mine monitoring system have been greatly improved compared with the old methods of warning, but there is still a big problem, and the problem of playing delay in the picture is very serious in the work. It can be found from the display that when the movement of the staff is small or slow, the picture is difficult to play normally, showing the overall outline of the staff, which is very obvious in the current period, as shown in figure 2.

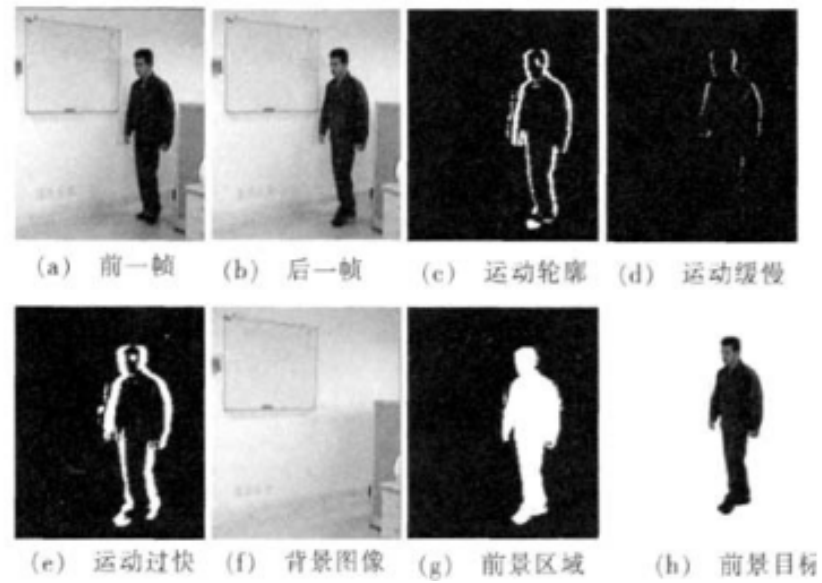

Figure 2 Monitoring images of traditional monitoring and testing systems

There are still many problems in the monitoring and testing system. If the operator moves quickly, the body will show a void on the display, and these problems must be solved as soon as possible, otherwise it will directly affect the working process. In addition, it is found that the system will not only be affected by the staff's action range, but also by the external environment, which makes it difficult for the monitor to understand the underground operation well, and the safety analysis work cannot be carried out smoothly.

\section{Target Detection Algorithm}

After understanding this situation, the system content can be supplemented flexibly, considering that the background difference method has the defect which affects the performance effect of the detection work, it is necessary to further optimize the algorithm, which can ensure the accuracy and accuracy of the numerical value of the algorithm, at the same time, the detection method should also 
be adjusted, the target contour extraction method should be used to detect the powerful grip of the underground construction environment, according to the previous inspection work, the situation of the empty image should be filled, the background model based on the color gradient should be established, the target monitoring effect can be improved, the safety probability of the safety accident can be reduced by using the background gradient extraction and the two contour models, and the result of the system calculation ability can be improved.

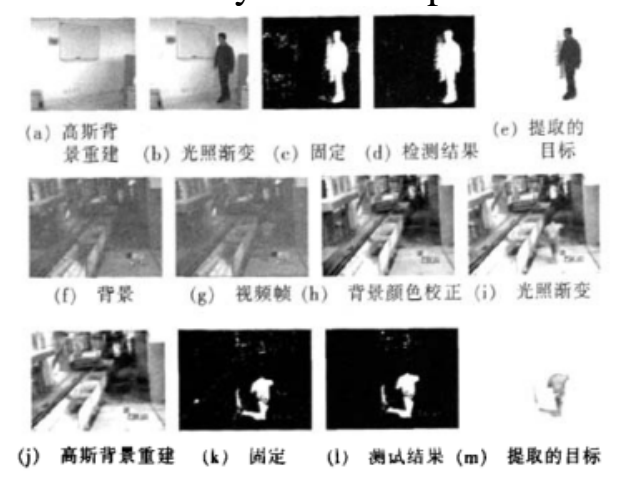

Figure 3 Image display effect of target extraction in light gradient process

It is also necessary to further understand the detection content and the degree of change of the target in the light slowly changing environment. The establishment of the Gao Si model in the detection system can greatly improve the accuracy of the calculation results. In the process of constructing the model using $\mathrm{K}$ Gaussian distribution, there is still a lot to pay attention to, timely online update, and it is very important to understand the representation background gradient. To study the system, we need to determine the working environment, and should know other work information, the $\mathrm{K}$ value selection needs to consider many factors, the value is too small, it will affect the system background generation effect, setting the $\mathrm{K}$ value too large will seriously affect the difficulty of the work, although it can deal with the more complex environment, but the followup work will become extremely difficult, and this study will position the $\mathrm{K}$ value 7 , so that the various elements can be well balanced. In addition, we should take into account that the matching link of the system algorithm will occupy a lot of time, we can use the approximate algorithm, in addition, we should design the parameters in the Gaussian distribution reasonably, so as to ensure that the model construction is in accordance with the actual situation. After setting the gaussian parameters in this study, the weight increment of the gaussian model is further accurate, so it is difficult for the traditional system to collect the target information in a short time, and will be disturbed by the moving target, which will affect the detection effect, and the underground working environment is very special, and will be disturbed by the external factors.

After analysis, it is not difficult to find that the moving target detection algorithm is really better than the traditional detection system. In the underground complex and changeable environment, the use of moving target detection algorithm can track the work of the staff and ensure the personal safety of the staff.

\section{Conclusion}

In order to improve the safety performance of downhole operation, it is necessary to enhance the safety consciousness of the workers. Mine safety monitoring, the use of moving target detection algorithm, can effectively detect the activity of workers in the underground, the underground environment has the characteristics of slow line change, so no doubt to a great extent improve the performance of the inspection work, this study to build a work model, after the study, proved that the motion target detection algorithm has a good pay Oh effect, and meet the comprehensive monitoring requirements of inclined roadway, the monitoring system using the motion target detection algorithm is simple, technical function rich, reasonable structure, which is an important guarantee for the coal mine operation to proceed smoothly. 


\section{Acknowledgements}

Research project of Gansu Higher Education Institute, project number: 2018C-25.

\section{References}

[1] Zheng, Pu., Bai, Hongyang., Li, Zhengmao., Guo, Hongwei. Design of accurate detection and tracking algorithm for moving targets under jitter interference. Journal of Instrumentation, pp. 1-10.

[2] Wang, Wei., Wang, Xiaopeng., Liang, Jincheng. Modified Vibe algorithm based on adaptive detection of moving objects .Journal of Measurement Science and Instrumentation, pp. 1-11.

[3] Zhao, Qian. Study on video monitoring image processing of coal mine underground dynamic target. Xi'an University of Science and Technology, 2014.

[4] He, Qian. Study on motion monitoring method of underground belt conveyor based on vision technology. Xi'an University of Science and Technology, 2012.

[5] Li, Dan., Qian, Jiansheng., Chai, Yanli. Research on moving target detection algorithm in mine safety monitoring. Television Technologies, vol. 33, no. 11, pp. 97-99. 